\title{
Influencing Factors and Correlation of the Degree of Intensive Care Unit Nurses' Perceived Job Stressors
}

\author{
Xiaohuan $\mathrm{Ma}^{1}$, Hong Guan ${ }^{2,}$, , Shu $\mathrm{Han}^{3}, \mathrm{Na} \mathrm{Li}^{4}$, Wenping $\mathrm{Mo}^{4}$ \\ ${ }^{1}$ ICU, Second People's Hospital, Dalian, China \\ ${ }^{2}$ Department of Nursing, Second Hospital, Dalian Medical University, Dalian, China \\ ${ }^{3}$ Department of Nursing, Second People's Hospital, Dalian, China \\ ${ }^{4}$ School of Nursing, Dalian Medical University, Dalian, China
}

Email address:

Liuhongguanhong@163.com (Hong Guan)

${ }^{*}$ Corresponding author

\section{To cite this article:}

Xiaohuan Ma, Hong Guan, Shu Han, Na Li, Wenping Mo. Influencing Factors and Correlation of the Degree of Intensive Care Unit Nurses' Perceived Job Stressors. American Journal of Nursing Science. Vol. 7, No. 4, 2018, pp. 152-158. doi: 10.11648/j.ajns.20180704.17

Received: June 5, 2018; Accepted: June 21, 2018; Published: July 13, 2018

\begin{abstract}
To identify the main job stressors of ICU nurses, and analyze their causes, influencing factors and correlation. Application of current status investigation methods, general information and the scale of "Chinese Nurse Stress Source Scale" of 301 ICU nurses at seven class A grade III general hospitals in Dalian were investigated and analyzed. The scores of "frequent shifts" are (3.35 \pm 1.00$)$, the scores of "too much work" are $(2.87 \pm 1.06)$, and the scores of "worry about mistakes in work" are $(2.85 \pm 1.06)$; Multiple linear regression analysis shows that education, working years in ICU, and average monthly salary are the main influencing factors that affect the nursing and work dimension $(P<0.05), R^{2}=0.148$. Age, working years in ICU, and average monthly salary are the main influencing factors that affect the time allocation and workload dimension $(P<0.05)$, $R^{2}=0.101$. Working years in ICU is the main influencing factor of the patient care dimension $(P<0.05), R^{2}=0.022$; The result of Spearman correlation analysis shows that the degree of perceived job stressors and influencing factors have a significant correlation $(P<0.05)$. The study shows that "Frequent shifts", "too much work", and "worry about mistakes in work" are the main job stressors, "age", "education", "working years in ICU”, and "average monthly salary" are the main influencing factors, there is significant correlation between the degree of perceived job stressors and influencing factors.
\end{abstract}

Keywords: ICU Nurse, Job Stressor, Influencing Factor, Correlation

\section{Introduction}

Stress is a state of reaction, is the result of interaction between human and environment, and is a relationship between people and environment. When environment changes beyond people's coping ability, will cause individuals to produce physical or psychological changes [1]. The modern stress viewpoints hold that stress is a kind of physical and mental tension, which is stimulated by internal and external environment stimulation, and the social, psychological, or biological stimuli are called stress sources, which threaten and challenge the survival of individuals [2].

Intensive care unit (ICU) is a modern medical technology, have a large number of advanced medical equipment, receive patients with critical conditions, and doctors and family members of the expectations of nurses are higher. Due to their environment and special rescue tasks, ICU nurse are the main caregivers, providing patients with good first aid and treatment [3]. Most patients in ICU are filled with various types of pipes. Facial expression of pain, painful cries and groans, and the noise of the instruments make nurses in depressed working environment for long time. A large number of domestic and international studies have shown that [4-6], due to ICU patients critically ill, many emergencies, advanced and complex equipment, and the various operational requirements, nurses are highly nervous and in a state of high stress.

In this study, we investigated the degree of perceived job stressors of ICU nurses in class A grade III general hospitals in Dalian city, analyzed the main influencing factors and correlations of stressors, and will provide scientific reference 
for nursing managers to reduce job stressors.

\section{Materials and Experiments}

\subsection{Materials}

The questionnaire survey was conducted between April 2016 and May 2016, in 301 ICU nurses at seven class A grade III general hospitals in Dalian. Inclusion criteria: Nurses working in ICU of class A grade III general hospitals in Dalian; Informed consent, voluntary participation; Exclusion criteria: Rotating nurses; Refresher nurses; Nurses in pregnancy, lactation, sickness, or maternity.

\subsection{Experiments}

\subsubsection{Research Tools}

Self-designed general information questionnaire, includes gender, age, education, working years in ICU, average monthly salary; "Chinese Nurse Stress Source Scale" includes 35 items, five dimensions, which are nursing and work (items 1 to 7), time allocation and workload (items 8 to 12), working environment and equipment (items 13 to 15 ), patient care (items 16 to 26), and management and interpersonal (items 27 to 35). Using a 4-point Likert-type method, "no" is scored at 0 point, "very light" notes 1 , "medium" notes 2," emphasis" notes 3, "serious" notes 4;
1.00 2.00 is mild stress, 2.01 3.00 is moderate stress, $3.01 \sim$ 4.00 is severe stress, higher scores indicate heavier stress. [7]

\subsubsection{Research Process}

ICU nurses were surveyed on-site, and questionnaires were recovered on-site. A total of 310 questionnaires were issued, 310 questionnaires were recovered, 301 questionnaires were valid, and the recovery rate was $100 \%$, the effective rate was $97 \%$.

\subsubsection{Statistical Analysis Methods}

The data is analyzed by SPSS 22.0 statistic software, and statistical analysis methods such as variance analysis, multiple linear regression analysis and Spearman correlation analysis were used.

\section{Result}

\subsection{General Information}

Among the 301 ICU nurses, there are 100 persons, whose age are 30 years and above, accounted for $33.2 \%$, there are 208 persons, whose education are bachelor and above, accounted for $69.1 \%$, there are 136 persons, whose working years in ICU are 5 and above, accounted for $45.2 \%$, and there are 148 persons, whose average monthly salary are 5,000 $¥$ and above, accounted for $49.2 \%$, other general information is shown in table 1 .

Table 1. General information of ICU nurses $(n=301)$.

\begin{tabular}{|c|c|c|c|c|c|}
\hline item & n & $\%$ & item & n & $\%$ \\
\hline gender & & & working years in ICU (years) & & \\
\hline male & 27 & 9 & $<1$ & 33 & 11.0 \\
\hline female & 274 & 91 & $1 \sim 2$ & 48 & 15.9 \\
\hline age(years) & & & $3 \sim 4$ & 84 & 27.9 \\
\hline$<20$ & 1 & 0.3 & $5 \sim 6$ & 58 & 19.3 \\
\hline $20 \sim 24$ & 43 & 14.3 & $6 \sim 8$ & 32 & 10.6 \\
\hline $25 \sim 29$ & 157 & 52.2 & $>8$ & 46 & 15.3 \\
\hline$>35$ & 23 & 7.6 & $1500 \sim 2000$ & 13 & 4.3 \\
\hline education & & & $2001 \sim 3000$ & 55 & 18.3 \\
\hline technical secondary school & 8 & 2.7 & $3001 \sim 5000$ & 80 & 26.6 \\
\hline junior college & 85 & 28.2 & $>5000$ & 153 & 50.8 \\
\hline bachelor & 204 & 67.8 & & & \\
\hline master or above & 4 & 1.3 & & & \\
\hline
\end{tabular}

\subsection{Degree of Perceived Job Stressors}

The degree of perceived stressor $\geq 2$ points, is a medium and severe stressor, there are 17 items, the average scores are ranked in descending order, shown in table 2.

Table 2. ICU nurse's perceived medium and severe stressors $(n=301)$.

\begin{tabular}{|c|c|c|c|c|c|}
\hline order & item & $x \pm s$ (score) & order & item & $x \pm s$ (score) \\
\hline 1 & frequent shifts & $3.35 \pm 1.00$ & 10 & nurses' work not recognized by patients and their families & $2.48 \pm 1.14$ \\
\hline 2 & too much work & $2.87 \pm 1.06$ & 11 & non-caring work too much & $2.47 \pm 1.19$ \\
\hline 3 & worry about mistakes in work & $2.87 \pm 1.06$ & 12 & patients don't cooperate & $2.45 \pm 1.09$ \\
\hline 4 & patients in care are more serious & $2.80 \pm 1.00$ & 13 & fewer opportunities for promotion & $2.43 \pm 1.04$ \\
\hline 5 & low wages and other benefits & $2.77 \pm 1.00$ & 14 & rude family & $2.28 \pm 1.00$ \\
\hline 6 & social status of nursing work is lower & $2.70 \pm 1.04$ & 15 & too much demanding in patients & $2.22 \pm 1.00$ \\
\hline 7 & fewer nurses at work & $2.64 \pm 1.06$ & 16 & no time for psychological care & $2.13 \pm 1.18$ \\
\hline 8 & fewer opportunities for further study & $2.53 \pm 1.26$ & 17 & rude patients & $2.08 \pm 0.99$ \\
\hline
\end{tabular}

\subsection{Distribution of Moderate and Severe Stressors in Different Dimensions}

ICU nurses 'moderate and severe stressors are concentrated in nursing and work, time allocation and workload, and patient 
care dimensions, shown in table 3.

Table 3. Distribution of middle and severe stressors in 3 dimensions $(n=301)$.

\begin{tabular}{lll}
\hline nursing and work & time allocation and workload & patient care \\
\hline frequent shifts & too much work & worry about mistakes in work \\
low wages and other benefits & fewer nurses at work & patients in care are more serious \\
lower social status of nursing work & more useless paperwork & nurses' work not recognized by patients and their families \\
fewer opportunities for further study & non-caring work too much & patients don't cooperate \\
fewer opportunities for promotion & no time for psychological care & $\begin{array}{l}\text { rude family } \\
\text { too much demanding in patient } \\
\end{array}$ \\
& & rude patient \\
\hline
\end{tabular}

\subsection{Univariate Analysis of Each Dimension of Moderate and Severe Stressors}

Age, education, working years in ICU, and average monthly salary have statistical significance in the 3 dimensions $(P<0.05)$, shown in table 4.

Table 4. The univariate analysis of the 3 dimensions.

\begin{tabular}{|c|c|c|c|c|c|c|c|c|c|c|}
\hline \multirow{2}{*}{ dimension } & \multicolumn{2}{|c|}{ gender } & \multicolumn{2}{|l|}{ age } & \multicolumn{2}{|c|}{ education } & \multicolumn{2}{|c|}{ working years in ICU } & \multicolumn{2}{|c|}{$\overline{\text { average monthly salary }}$} \\
\hline & $F$ & $P$ & $F$ & $P$ & $F$ & $P$ & $F$ & $\boldsymbol{P}$ & $F$ & $P$ \\
\hline nursing and work & 0.76 & 0.38 & 1.41 & 0.23 & 5.16 & $0.00 *$ & 6.57 & $0.00 *$ & 2.65 & $0.04 *$ \\
\hline time allocation and workload & 0.64 & 0.43 & 2.71 & $0.03 *$ & 1.55 & 0.20 & 3.95 & $0.02 *$ & 5.54 & $0.01 *$ \\
\hline patient care & 0.00 & 0.97 & 1.28 & 0.28 & 0.42 & 0.74 & 3.02 & $0.01 *$ & 3.00 & $0.03 *$ \\
\hline
\end{tabular}

Note: * indicates $P<0.05$

\subsection{Multiple Linear Regression Analysis of Moderate and Severe Stressors}

In the multiple linear regression equation, the test standard is $\alpha=0.05$, the excluding standard is $\alpha=0.10$. Taking the three dimensions of moderate and severe stressors as dependent variables, and taking the factors $(P<0.05)$ in the univariate analysis of each dimension as independent variables, the regression model is established to perform multiple linear regression analysis. The assignment is detailed in table 5.

Table 5. The regression equation variable assignment.

\begin{tabular}{ll}
\hline variable & assignment \\
\hline Age (years) & $1=<20,2=20 \sim 24,3=25 \sim 29,4=30 \sim 35,5=>35$ \\
& $1=$ technical secondary school, $2=$ junior \\
education & college, $3=$ bachelor, $4=$ MA or above \\
working years in ICU (years) & $\begin{array}{l}1=<1,2=1 \sim 2,3=3 \sim 4,4=5 \sim 6,5=7 \sim 8,6=>8 \\
1=1500 \sim 2000,2=2001 \sim 3000,3=3001 \sim 4000, \\
\text { average monthly salary (¥) }\end{array}$ \\
\hline
\end{tabular}

The result of multiple linear regression analysis of nursing and work shows that the difference in nurses' stressors perception with different education, working years in ICU, and average monthly salary is statistically significant $(P<0.05)$, indicates education, working years in ICU, and average monthly salary are the main influencing factors; $R^{2}=0.148$, indicates the three influencing factors can explain the difference $14.8 \%$, shown in table 6 .

Table 6. Multiple linear regression analysis of nursing and work.

\begin{tabular}{llllll}
\hline variable & B & Se & Beta & $\boldsymbol{t}$ & $\boldsymbol{p}$ \\
\hline constant & 13.19 & 1.54 & & 8.55 & $0.00^{*}$ \\
education & -1.19 & 0.20 & -0.30 & -5.88 & $0.00^{*}$ \\
working years in ICU & 0.39 & 0.08 & 0.25 & 5.20 & $0.00^{*}$ \\
average monthly salary & -0.57 & 0.25 & -0.13 & -2.29 & $0.02 *$ \\
\hline
\end{tabular}

Note: $R^{2}=0.148, F=3.726$, *indicate $P<0.05$
The result of multiple linear regression analysis of time allocation and workload shows that the difference in nurses' stressors perception of different age, working years in ICU, and average monthly salary is statistically significant $(P<0.05)$, indicates age, working years in ICU, and average monthly salary are the main influencing factors; $R^{2}=0.101$, indicates the three influencing factors can explain the difference $10.1 \%$, shown in table 7.

Table 7. Multiple linear regression analysis of time allocation and workload.

\begin{tabular}{llllll}
\hline variable & B & Se & Beta & $\boldsymbol{t}$ & $\boldsymbol{p}$ \\
\hline constant & 10.95 & 1.78 & & 6.16 & $0.00^{*}$ \\
age & 0.83 & 0.42 & 0.15 & 1.98 & $0.04^{*}$ \\
working years in ICU & 0.48 & 0.22 & 0.16 & 2.19 & $0.03^{*}$ \\
average monthly salary & -1.01 & 0.29 & -0.20 & -3.52 & $0.00^{*}$ \\
\hline
\end{tabular}

Note: $R^{2}=0.101, F=8.330,{ }^{*}$ indicate $P<0.05$

The result of multiple linear regression analysis of patient care dimension shows that the difference in nurses' perceived stressors of different working years in ICU is statistically significant $(P<0.05)$, indicates working years in ICU is the main influencing factor; $R^{2}=0.022$, indicates it can explain the difference $2.2 \%$, shown in table 8 .

Table 8. Multiple linear regression analysis of patient care dimension.

\begin{tabular}{llllll}
\hline variable & B & Se & Beta & $\boldsymbol{t}$ & $\boldsymbol{p}$ \\
\hline constant & 16.47 & 2.16 & & 7.57 & $0.00^{*}$ \\
working years in ICU & 0.51 & 0.27 & 0.15 & 2.02 & $0.04^{*}$ \\
average monthly salary & -0.19 & 0.35 & -0.03 & -0.53 & 0.60 \\
\hline
\end{tabular}

Note: $R^{2}=0.022, F=3.590$, * indicate $P<0.05$ 


\subsection{Correlation Analysis Between the Degree of Perception and Influencing Factors of Moderate and Severe Stressors in ICU Nurses}

Spearman correlation analysis is applied to the three dimensions and their influencing factors. Nursing and work has a significant correlation with education and working years in ICU $(P<0.05)$; Time allocation and workload has a significant correlation with age, working years in ICU, and average monthly salary $(P<0.05)$; the correlation between patient care and working years in ICU is significant $(P<0.05)$, shown in table 9 .

Table 9. Correlation analysis of dimensional perception and influencing factors.

\begin{tabular}{llllll}
\hline & & age & education & working years in ICU & average monthly salary \\
\hline nursing and & $\mathrm{r}$ & & $-0.18^{*}$ & $0.21^{*}$ & -0.06 \\
work & $P$ & & 0.00 & 0.00 & 0.28 \\
time allocation & $\mathrm{r}$ & $0.24^{*}$ & & $0.21^{*}$ & $-0.14^{*}$ \\
and workload & $P$ & 0.00 & & 0.00 & 0.02 \\
patient care & $\mathrm{r}$ & & & $0.12^{*}$ & 0.04 \\
& $P$ & & & & \\
\hline
\end{tabular}

Note:*. Correlation is significant at 0.05 confidence level(bilateral)

\section{Discussion}

The Health and Safety Executive statistics shows that nurses are the 2nd most stressful career following the teachers [8], nurses' working stress is at a high level [9]. ICU patients are in critical condition and the full treatment without accompanying. Even though ICU nurses complete the complex treatment, nursing work, the family cannot correctly understand the hard work, all these have increased ICU nurses psychological pressure and working stress [10]. The working stress of nurses has become a kind of occupational danger, too high stress, not only affect the physical and mental health of nurses, nurses on job satisfaction, nurse attendance and turnover rate, but also affect the quality of nursing service [11]. To reduce or eliminate the degree of perceived stressors, we should to clarify the main stressors and influencing factors and take reasonable and effective measures to reduce the controllable stressors, so as to further improve nurses' professional happiness, stabilize the nursing team, and ensure patient safety.

\subsection{The Major Stressors of ICU Nurses}

From table 2, the top three stressors are "frequent shifts", "too much work", and "worry about mistakes in work". The results are same as the 2012-2015 domestic scholar survey results [12-15], but slightly different in perception.

\subsubsection{Causative Analysis of "Frequent Shifts" as the Main Stressor}

"Frequent shifts" is a severe stressor, ranks first in the ICU nurse's stressors with a score of $(3.35 \pm 1.00)$. The nature of nursing working is to shift. If the ICU nurses understaffed, excessive night shift will make the nurses circadian clock disorder, diet and sleep without regularity, disrupt the normal physiological rhythms, affect physical and mental health, easy to produce anxiety, sleep disorders, so that nurses are often in a state of fatigue. China Health Industry Standards stipulate that the ratio of ICU nurses to actual beds should not be less than 3:1. Nurses understaffed will make nurses work overload and often in a state of fatigue [16], which is the main reason for "frequent shifts" become the main stressor.

\subsubsection{Causative Analysis of "Too Much Work" as the Main Stressor}

ICU nurses not only act as managers of daily medical activities, executors of the treatment process, but also as caregivers of patient's life, they often perform several duties [17]. Multiple roles often make nurses physically and mentally exhausted and powerless. Xiaomei Li et al [7] have fond that nurses feel tired at work as high as $59.1 \%$ in general departments. Dan Li et al [18] have fond ICU nurses have a higher sense of stress than clinic nurses, but work achievement is lower. ICU nurses' working stress and fatigue are significantly higher than other departments, and a high degree of working stress and fatigue will affect the physical and mental health, work ability, even cause nurses to quit their job. As a specialist nurse, the work should embody their professional value. Provide sufficient nurses for the ICU, scientifically and rationally distribute the workload of nurses, will can effectively reduce the nurses' tiredness, relieve the stress source perception and improve the working efficiency.

\subsubsection{Causative Analysis of "Worry About Mistakes in Work" as the Main Stressor}

The ICU patients' condition is critical, complex, and rapidly changing, nurses must master multidisciplinary rescue knowledge, and have a keen observation and agile thinking ability. A little carelessness may cause serious consequences, resulting in irreparable losses. High mortality rate of ICU patients, long-term noise pollution, and closed work space, can make nurses in a high degree of stress [11]. Domestic scholars [12] have found that the incidence of moderate and severe anxiety in ICU nurses is as high as $53.1 \%$. ICU nurses need to continuously improve skills and update knowledge. Learning stress, severe psychological stress, and high degree of working burnout, have adverse effects on nurses' physiology and psychology. If not giving timely solutions, errors and accidents are inevitable. Nursing is a maintenance of life and health, and is closely related to life. Once the accident occurs, may bring irreparable harm to the patient, and cause the nurse has a heavy sense of guilt. Improving ICU nurses' working environment, reducing working stress and strengthening the cultivation of psychological capital, are 
important factors to promote the scientific development of professional nurses' career [19].

Paying attention to the psychological health of ICU nurses, setting up a mental health counseling team, providing psychological counseling, encouraging nurses to communicate with colleagues and managers, will reduce the chance of errors and accidents. If the accident happens, timely analysis of the causes, take practical measures to avoid the occurrence of similar incidents, and the nurses to give psychological treatment, can reduce the psychological negative emotions, make nurses subsequent work easily.

\subsection{Analysis of Job Stressors Characteristics of ICU Nurses}

\subsubsection{Educational Differences}

Multiple linear regression analysis in table 6 shows that ICU nurses with different education have statistical significance in the nursing and work dimension $(P=0.00)$. "Education" has a Beta value of -0.30 , indicating that the degree of dimensional perception increases by -0.30 points for each stage of education advancement. The result of Spearman correlation analysis of "education" and perception of the nursing and work dimension is $P=0.01, R=-0.18$, which shows that the perceptual degree is negatively correlated with it, and it can relieve the nursing and working dimension pressure source perception by elevating the ICU nurse's degree.

The moderate and severe stressors perceived in the nursing and work dimension include "frequent shifts", "low wages and other benefits", "lower social status in nursing work", and "fewer opportunities for further studies", "fewer opportunities for promotion". Foreign scholars [20] also have found that lower status, fewer opportunity of promotion and going out to study are the main stressors of ICU nurses.

Improving nurses' psychological endurance can improve nurses' working enthusiasm [21], and to relieve working stress 。 In this study, there are 208 bachelor's degrees and above, the proportion is $69.1 \%$. Education not only reflects a person's educational level, learning ability, but also has a closed relationship with work ability and psychological endurance. When the stress acts on the body, it generates stress or not, depending on the individual's knowledge level and coping style. Highly educated nurses have a stronger ability to find and understand problems in the work. Through clinical practice, they are more able to recognize the adverse effects of "frequent shifts" on the physiology and psychology of nurses. At the same time, in this special work environment such as ICU, nurses with higher degree will faster discover problems, and have stronger ability to solve them. Therefore, when the same stressors are applied to nurses with relatively high academic qualifications, they will take a more positive approach to solve problems and will reduce the perceived stressor.

The professional benefits of nurses can be improved by obtaining specialized certificates, improving professional titles, and participating in clinical teaching [22]. Strengthening the training of specialized knowledge and ability, giving more chances for nurses to further study, paying more attention to the continuous education, strengthening the training professional skills in critical care, improving the technical level, and meeting their needs for recognition and promotion, can not only improve the quality of nursing services, but also enable them to obtain the approval of colleagues, patients, and families in their work, mitigate perceptions of "low wages and other benefits", "lower social status in nursing work", and "fewer opportunities for further studies", "fewer opportunities for promotion".

\subsubsection{Age Difference}

The multiple linear regression results in table 7 show that ICU nurses with different ages have statistical difference in time allocation and workload dimension $(P=0.04)$, and "age" has a Beta value of 0.15 , indicating that in each stage of 5 years of age, the dimensional perception of stressors increase by 0.15 . The result of Spearman correlation analysis of "age" and perception of the time allocation and workload dimension is $P=0.00, R=0.24$, it shows that the perception degree is positively correlated with age, which indicates that ICU nurses have increased the sense of stressors in time allocation and workload dimension with the increase of age.

ICU nurses' perception of moderate and severe stressors in time allocation and workload dimension include "too much work", "fewer nurses at work", "more useless paperwork", "non-caring work too much", and "no time for psychological care"

A total of 301 ICU nurses surveyed, 100 of them aged 30 and over, accounted for $33.2 \%$. They are the backbone of ICU, but also the main body to bear the family responsibility. The dual responsibilities will aggravate the nurses' physical and psychological stress. At the same time, age to a certain extent reflects the working life in ICU. ICU nurses have long been in a tense and high-stress state, and work fatigue is heavy, all those making "fewer nurses at work" and "too much work" as medium and severe stressors. Nursing is a practical science, as the working years increases, nurses are more able to find "more useless paperwork" in their work. In recent years, our country advocates to liberate nurses from nursing documents and give more time to patients. In this study, the "more useless paperwork" is a moderate stress, score is $(2.50 \pm 1.08)$, which is slightly lower than the survey score of domestic scholars from 2012 to 2015. Patients isolated from family, multiple roles to play, make "non-caring work too much" become the main stressor for ICU nurses. A large number of domestic and international investigations and studies have shown that [23-25], while ICU patients received good treatment conditions, they also have a significant impact on their physiology and psychology, the incidence of anxiety is $46 \%-66 \%$, which seriously affects the quality of life of patients and their families. With the increase of working years, ICU nurses are more and more aware of the importance of psychological nursing to ICU patients and their families, making "no time for psychological care" become a moderate stressor.

Strengthening the skills training of young nurses in critical 
condition, can effectively relieve work stress of senior nurses. At the same time, the ICU is equipped with enough specialist nurses to liberate nurses from tedious tasks and make their work more professional. All those can effectively reduce the perception of "too much work", "fewer nurses at work", "more useless paperwork", and "non-caring work too much", and nurses can have more time to pay attention to the patient's mental health, alleviate the problem of "no time for psychological care".

\subsubsection{Differences in Working Years in ICU}

Multiple linear regression analysis in table 6 shows that nurses with different working years in ICU has statistically significant difference in nursing and work dimension $(P=$ 0.00 ), and the Beta value is 0.25 , it shows that the sensitivity of the stress dimension increases by 0.25 points when ICU nurse's working life increase by 2 years. In the Spearman correlation analysis of "working years in ICU" and perception of the nursing and work dimension, the result is $P=0.00, R=$ 0.21 , it shows that the perception degree is positively correlated with working life, which indicates that ICU nurses have increased the sense of stressors in nursing and work dimension with the increasing of working life.

Multiple linear regression analysis in table 7 shows that nurses with different working years in ICU have a statistically significant difference in time allocation and workload dimension $(P=0.03)$, and the Beta value is 0.16 , it shows that the sensitivity of the stress dimension increases by 0.16 points when ICU nurse's working life increase by 2 years. In the Spearman correlation analysis of "working years in ICU" and the perception of time allocation and workload dimension, the result is $P=0.00, R=0.21$, indicating that with the growth of ICU working years, nurses 'perception gradually increase, and significantly positive correlation exist.

Multiple linear regression analysis in table 8 shows that nurses with different working years in ICU have a statistically significant difference in patient care dimension $(P=0.04)$, and the Beta value is 0.15 , it shows that the sensitivity of stress dimension increases by 0.15 when ICU nurse's working life increase by 2 years. In the Spearman-related analysis of "working years in ICU" and the perception of patient care, the result is $P=0.04, R=0.12$, indicating that significantly positive correlation exist, and with the growth of ICU working years, the nurses 'perception aggravate.

Working years in ICU is the main influencing factor of 17 items of ICU nurses' moderate and severe stressors. ICU is the focal point of critically ill patients, patient's condition changes rapidly, more sudden events, high standards of work requirements, and high-intensity workload, etc., which make nurses working stress is significantly higher than those in ordinary wards [26]. If nurses work in high-stress environment for long time, it would have adverse effects on their psychology and physiology, and would aggravate the perceived degree of stressors. In this study, the number of nurses who work in ICU for 5 years and above accounted for 136, and the proportion is $45.2 \%$. They are the ICU middle force, the backbone of the department, and the main force of the first line of work, directly facing various stress, but also responsible for the training and teaching work of new nurses, all these will make ICU working life long nurses feel high stress.

Staffed ICU with sufficient clinical experience and skill nurses will speed up the training of ICU specialist nurses and improve the quality of ICU nursing. We will strengthen the selection and training of the ICU backbone nurses with a certain number of years, ability of emergency and disease observation, problem-solving skills, then provide them more opportunities for further study. So that young nurses realize through their continuous efforts, the hospital is willing to provide more opportunities, for promoting the active work, improving their sense of responsibility and self-confidence, and relieving the sense of stressors.

\subsubsection{Differences in Average Monthly Salary}

Multiple linear regression analysis in table 6 shows that ICU nurses with different average monthly salary have a statistically significant difference in nursing and work dimension $(P=0.02)$, and the "average monthly salary" has a Beta value of -2.29 , it shows that the average monthly salary increases by $1000 ¥$, and the degree of perception decreases by 2.29 points.

Multiple linear regression analysis in table 7 shows that ICU nurses with different average monthly salary have statistical significance in time allocation and workload dimension $(P=0.00)$, and "average monthly salary" has a Beta value of -3.52 , it shows that the average monthly salary increases by $1000 ¥$, the degree of perception decreases by3.52 points. In the Spearman correlation analysis of "average monthly salary" and the perception of time allocation and workload dimension, the result is $P=0.02, R=$ -0.14 , indicating there is a significantly negatively correlation exist, and with the average monthly salary increasing, the degree of stressor perception is alleviated.

In the 301 investigators, 148 ICU nurses are paid less than $5,000 ¥$, accounting for $49.2 \%$. Because the ICU work environment is special, such as heavy workload, insufficient personnel, and multiple roles, make the psychological stress and the burnout feeling increasing, causes the nurses to perceive the pay and the return is disproportionate, easily produces the negative emotion, aggravates the stressors perception degree in the work.

As a special department, the material and spirit reward mechanism should be established to improve the treatment of ICU nurses. At the same time, the manager should staff enough nurses, allocate workload scientifically and reasonably, fully mobilize the nurses' enthusiasm, and meet their recognition, education, promotion needs. The reward of material and spirit is the affirmation of the work ability, not only make the nurse realize the pride of being a professional, but also is a kind of encouragement means, make the nurse feel labor have income, raise his professional sense of belonging [10].

\section{Conclusion}

This study shows that "Frequent shift, "too much work", 
and "worry about mistakes in work " are the main job stressors of ICU nurses in class A grade III general hospital, and "age", "education", "working years in ICU", and "average monthly salary" are the main influencing factors. Managers from the above aspects to take measures to staff ICU with sufficient specialized nurses, to establish the reasonable compensation system, to perfect their welfare benefits, to meet their needs for further study, promotion, so as to improve their working environment, and to relieve working stress.

\section{Limitations and Improvements}

\subsection{Limitations}

(1) Restricted by the region, this study only investigated the comprehensive ICU nurses of class A grade III general hospitals in Dalian City, and the representative is limited.

(2) This study is a cross-sectional investigation, and the job stressors in different periods of the same nurses need further study.

\subsection{Improvements}

(1) To increase ICU nurses in different regions and different grade hospitals.

(2) To further study on the perception of stressors in different periods of the same nurses in ICU.

\section{References}

[1] Lazarus RS. Emotions and interpersonal relationships: toward a person-centreed conceptualization of emotions and coping $[\mathrm{J}]$ Pers. 2006, 74(1):9-46.

[2] Wenhao Yue, Hongjing Zhang, Fang Pan. Medical Psychology [M]. Beijing: Science Press, 2003:118-121.

[3] Hewitt J. Psycho-affective disorder in intensive care unit: a review [J]. Clin Nurs, 2002, 11(5):575-584.

[4] Yingchao Liu. Influencing factors and intervention measures of ICU nurses in intensive care unit [J]. China Old Age Medical Insurance 2012, 10(2)71-72.

[5] Ward L. Mental health nursing and stress: Maintaining balance [J]. Int J Ment Health Nurs, 2011, 20(2):77-85.

[6] Adriaenssens J, De Gucht V, Van Der Doef M, et al. Exploring the burden of emergency care: Predictors of stress-health outcomes in emergency nurses [J]. Adv Nurs, 2011, 104(10):1365-1368.

[7] Xiaomei Li, Yanjun Liu. Investigation of work stress sources and job fatigue among nurses [J]. Chinese Journal of Nursing, 2000, 35(11):645-646.

[8] Hoban V. How to management stress [J]. Nursing Time. 2004, 100(2):64.

[9] Yang Liu. Survey on the working pressure of nurses in a classified 3A hospital in Qiqihaer city [D]. Jilin University, 2016.
[10] Yujuan Li. Investigation and analysis of job stress and job burnout of nurses in intensive care unit [J]. Journal of Qiqihar Medical University, 2016(37):1107-1108.

[11] Xiaolin Li, Lan Bai. Stress Source Analysis and Response Measures for Nurses in ICU [J]. People's Liberation Army Nursing Magazine, 2007, (2):49-51.

[12] Xiaoling Huang, Wei Lin. ICU nurse stress source and anxiety situation investigation and analysis [J]. Fujian Medical Magazine, 2014, 34(2):124-127.

[13] Hongyun Wei, Yulin Gao, Xueling Yang, et al. 439 ICU nurses work stress level and related factors analysis [J]. Journal of Nursing, 2014, (12):65-68.

[14] Juan Sun, Junxiu Zhang, Tusheng Chen. Stress source analysis and response studies of nurses in ICU [J]. Qilu Nursing Magazine, 2015, (6):57-59.

[15] Congcong Liu. The Status and Influencing Factors of Nurses' Work Engagement in a classified 3A General Hospital [D]. Shandong University, 2015.

[16] Wenjuan Qiu, Dong Liu, Yulan Feng, et al. ICU nurse occupational pressure source analysis and countermeasures $[\mathrm{J}]$. People's Liberation Army Hospital Management Magazine, 2013, (8): 781-783.

[17] Xiaohua Tian, Changxiang Chen. Correlation analysis of professional stress and job burnout in ICU nurses [J]. Nursing Practice and Research, 2014, 11: 14-15.

[18] Dan Li, Wangjian Li, Lili Tang, et al. ICU Nurse's work pressure source, fatigue, and blood pressure, blood sugar, and blood lipid changes[J]. People's Liberation Army Nursing Magazine, 2012, 29(4A).

[19] Xuebing Jing, Hong Liu, Xiaojie Fang, et al. Study on the relationship between job stressors, psychological capital and professional career of critical specialized nurses [J]. Chinese Nursing Research, 2016, 30(9)3348-3351.

[20] Melanie V, Paolo M, Thomas P, et al. Burnout in a surgical ICU team [J]. Intensive Care Med, 2008, 34: 152-156.

[21] Shuping Zhang. Correlation between nurses' psychological endurance and job stressors [J]. Chinese Nursing Research, 2017, 31(9): 1124-1126.

[22] Yonghong Wang. Study on the status and influencing factors of occupational benefits of nurses in a classified 3A hospital in Shijiazhuang city [D]. Hebei Medical University, 2017.

[23] Stevenson JE, Colantuoni E, Bienvenu OJ, et a1. General anxiety symptom after acute lung injury [J]. Journal of Psychological Research, 2013, 75: 287-293.

[24] Hua Lin, Shuhong Gao, Fangfang Song, et al. Investigation and analysis of psychological pressure sources in awake patients of ICU [J]. Chinese Journal of Modern Nursing, 2015, 21(25):3038-3040.

[25] Qiufang Gao, Haiyan Xing, Yunbo Sun, et al. Anxiety status of ICU patients and its related factors[ J]. Advances in modern biomedicine, 2012, 12(15): 931-2933.

[26] Yuan $\mathrm{Xu}$, Xinjuan Wu. Research status and application of psychological contract of nursing staff [J]. The Chinese Journal of Nursing, 2012, 47(5): 476-478. 